ISSN: 2600-5859

WwW.concienciadigital.org

Vol. 3, $\mathrm{N}^{\circ} 1.2$, p. 6-19, marzo, 2020

\title{
Usos y beneficios de las aplicaciones móviles en las empresas de la ciudad de Riobamba
}

\section{Uses and benefits of mobile applications in business in the city of Riobamba}

\author{
Jimena Catalina Viteri Ojeda. ${ }^{1}$, Carmita Efigenia Andrade Álvarez. ${ }^{2}$, Nakira Pamela Valencia
} Ortiz. ${ }^{3} \&$ Christian Andrés Castro Viteri. ${ }^{4}$

Recibido:05-01-2020 / Revisado: 22-01-2020 /Aceptado: 03-02-2020/ Publicado: 05-03-2020

\begin{abstract}
.
DOI: $\underline{\text { https://doi.org/10.33262/concienciadigital.v3i1.2.1165 }}$

The technological changes that have taken place worldwide in various areas in recent decades are evident, and the business world is no exception. Access to new information and communication technologies has allowed a dynamic interaction between the company-client relationships. It is especially in the field of versatility concerning the exponential increase in the use of mobile smart devices, tablets, cell phones facilitating the use of mobile applications, these have now become highly supportive tools for performing routine processes and tasks. In this document, the best benefits of the use of the most common mobile applications used in public and private companies in the city of Riobamba were determined, both for companies and for customers or users. An exploratory methodology was used with a probabilistic sample through a simple random sampling. The main global trends are evidenced in the results obtained through the surveys. It should be noted that there are wide use and preference in the use of applications in non-business versions. However, these tools foster customer loyalty and a sense of belonging on the part of company staff, while facilitating internal communication in organizations.

${ }^{1}$ Escuela Superior Politécnica de Chimborazo, Facultad de Administración de Empresas, Carrera de Administración de Empresa. Riobamba, Ecuador, jcviteri@espoch.edu.ec

${ }^{2}$ Escuela Superior Politécnica de Chimborazo, Facultad de Administración de Empresas, Carrera de Administración de Empresa. Riobamba, Ecuador, candrade_a@espoch.edu.ec

${ }^{3}$ Escuela Superior Politécnica de Chimborazo, Facultad de Administración de Empresas, Carrera de Administración de Empresa. Riobamba, Ecuador, nakira.valencia@espoch.edu.ec

${ }^{4}$ Gobierno Autónomo Descentralizado Municipal de Archidona, Departamento de Desarrollo Social. Archidona, Ecuador, christrian.castro@archidona.gob.ec
\end{abstract}


Keywords: Computer application, Company, Information technology, Computation, Comunication.

\section{Resumen.}

En evidente los cambios tecnológicos que se han dado a nivel mundial en diversas áreas en las últimas décadas, y el mundo empresarial no es la excepción. El acceso a las nuevas tecnologías de información y comunicación ha permitido una interacción dinámica entre la relación empresacliente, especialmente en el ámbito de versatilidad respecto al incremento exponencial en el uso de los dispositivos inteligentes móviles, tablets, celulares facilitando el empleo de las aplicaciones móviles, las actualmente se han convertido en herramientas de gran apoyo para la realización de procesos y tareas rutinarias. En el presente escrito se determinaron cuáles son los mejores beneficios que brindan el empleo de aplicaciones móviles más comunes utilizadas en las empresas públicas y privadas de la ciudad de Riobamba, tanto para las empresas como para los clientes o usuarios; para ello se utilizó una metodología exploratoria con una muestra probabilística a través de un muestreo aleatorio simple. Las principales tendencias mundiales se ponen en evidencia en los resultados obtenidos a través de las encuestas. Cabe recalcar que existe una amplia utilización y preferencia en el uso de aplicaciones en las versiones no empresariales; sin embargo, estas herramientas fomentan la fidelidad por parte de los clientes y el sentido de pertenencia por parte del personal de las empresas, a la vez que facilita la comunicación interna en las organizaciones.

Palabras claves: Aplicación informática, Empresa, Tecnología de la comunicación, Computación, Comunicación.

\section{Introducción.}

El acelerado crecimiento y desarrollo tecnológico en las últimas décadas ha cambiado radicalmente la forma de relacionarse a nivel personal, empresarial e incluso entre las sociedades. El contar con medios que facilitan la posibilidad de combinar la interpretación técnica con estrategias empresariales, políticas y estatales, facilita una mayor productividad e igualdad de oportunidades, fomentado la inter e intra relación entre empresas y/o clientes. (CEPAL, 2018).

La movilidad es la base de la comunicación actual, esto se debe principalmente a tres factores que aunque el uno no depende el otro se encuentran relacionados. El primero, al gran incremento en el uso de celulares, ipad, segundo la facilidad que tienen que ahora la mayor parte de las personas, en especial aquellas que viven en el sector urbano, para acceder a una interconexión de internet y tercero el auge en el uso de las aplicaciones móviles de mensajería instantánea y de las redes sociales (Nicolini \& Parodi, 2015). El empleo de las herramientas tecnológicas sin barreras de edad, país, región o continente trae como consecuencia la estandarización de patrones de comportamiento a nivel universal (Arni Consulting Gruop, 2017). Son diversos los contextos en 
los que estas formas de comunicación se hacen presentes y tanto las personas como las empresas deben estar en la capacidad de adaptarse y desenvolverse de forma responsable y eficaz en las situaciones a las que se enfrenten (Rodríguez G., 2015).

Gracias a las Tecnologías de Información y Comunicación (TIC) es posible la deslocalización y virtualización del trabajo, lo que hace innecesario que las relaciones se realicen cara a cara, ya que se las pueden efectuar desde cualquier lugar y de forma eficiente, lo que ha propiciado algunas nuevas formas de organización del trabajo, como son: el teletrabajo, el outsourcing, la centralización y deslocalización de funciones de menor valor añadido, las formas de comunicaciones y reuniones no presenciales (Álvarez, 2011), así como las sesiones de trabajo virtuales que conllevan mayor eficiencia en lo referente a los costes y al acceso a la información, además el uso del correo electrónico desde dispositivos móviles, facilita la comunicación y la conectividad a la vez que reduce los desplazamientos innecesarios. (prnoticias, 2015)

En Ecuador es creciente el número de empresas y establecimientos, esto se evidencia en el índice de crecimiento presentado por el Instituto Nacional de Estadística y Censo (INEC), en su Directorio de Empresas y Establecimientos (DIEE) 2018, que, presenta un crecimiento del 1.15\% para el año 2017 con respecto al 2016, lo cual sumado a la existencia también de un incremento constante de acceso a internet desde diferentes ámbitos de la sociedad en donde se evidencia que la utilización más frecuente de las tecnologías de comunicación es para encontrar información y para comunicarse, así como el incremento en un $7.5 \%$ en la posesión de teléfonos inteligentes (ENEMDU_TIC,2017), ponen de manifiesto la importancia que tiene el uso de tecnologías como parte integral de la comunicación empresarial (Castastros RUC 2019).

Debido a la alta disponibilidad tecnológica, su utilización en las comunicaciones por parte de las pequeñas, medianas, y grandes empresas es inevitable; sin embargo, resulta complejo el balancear el trabajo y la familia, con el objetivo de lograr una mejor calidad de vida. (Sodexo, 2014). Uno de los principales beneficios de una comunicación de calidad es el sentimiento de pertinencia del empleado con respecto a la empresa y las pymes son cada vez más conscientes de que gracias a este tipo de comunicación se pueden conseguir efectos positivos para todas las partes; ya que, incluso ayudan a afianzar la fidelidad de los clientes (EmprendePyme.net, 2016). Otro aspecto importante a considera, es el establecimiento de canales formales de comunicación dentro de la empresa como son: aplicaciones de mensajería, correo electrónico, redes sociales, entre otros resulta vital para fomentar el compromiso y la participación de las personas que forman parte de la organización (Campos J., 2015).

Entre las herramientas de comunicación y difusión digitales se disponen de las webs móviles y de las app (aplicaciones móviles). La App, es una aplicación de software que se instala en dispositivos móviles o tablets para ayudar al usuario en una tarea definida; es decir, servir como asistente en operaciones y gestiones del día a día ya sea de carácter profesional, de ocio o entretenimiento. La web móvil es una página web adaptada a los formatos de los dispositivos móviles (CEUPE, 2019). 
Según estudios realizados un $80 \%$ de los usuarios en general prefieren el uso de aplicaciones móviles, esto se debe a varios factores como por ejemplo la sencillez de su aplicación, el que permitan obtener medidas de uso de acuerdo a las necesidades de una organización, pudiendo ser públicas o privadas (Jiménez, 2012); La Economía o en ocasiones nulidad al emplearlas porque muchas de ellas son aplicaciones gratis, el acceso inmediato que un usuario puede tener hacia esta, etc. (Ritacco V., 2015). Existen diversas aplicaciones utilizadas para el envío de mensajes y compartición de documentos que son utilizadas como parte de la comunicación integral en las empresas, entre las más importantes tenemos (Facchin J., 2019):

- WhatsApp: Es una de las aplicaciones móviles más utilizadas en el mundo, se caracteriza por su facilidad de uso y a inmediatez en la comunicación directa ya que permite enviar texto escrito, mensajes de voz, imágenes y videos de manera individual o a un grupo de personas.

- WhatsApp: Es una de las aplicaciones móviles más utilizadas en el mundo, se caracteriza por su facilidad de uso y a inmediatez en la comunicación directa ya que permite enviar texto escrito, mensajes de voz, imágenes y videos de manera individual o a un grupo de personas.

- WhatsApp Business : pensado en las pequeñas empresas y negocios para que puedan interactuar fácilmente con sus clientes mediante herramientas que les permiten automatizar, ordenar y responder mensajes de forma rápida. Los usuarios pueden visualizar en todo momento datos empresariales como geolocalización, horarios, y dejar quejas o sugerencias.

- Facebook: Esta red social permite múltiples acciones para conseguir la creación de una cuenta bien orientada hacia los objetivos empresariales. Ya que desde su App para páginas de empresas llamada Facebook Pages, se puede crear una comunidad, ofrecer un producto o servicio orientado hacia un público específico, así como realizar un análisis profundo del comportamiento del mercado y los seguidores.

- Telegram: Es una herramienta muy útil para empresarios y emprendedores ya que permite la creación de grupos temáticos y muy segmentados entre personas con aficiones o profesionales afines, sin necesidad de que estos sepan los números de teléfono unos de los otros por lo que se mantiene cierta privacidad en las conversaciones.

- Hibox: Es un servicio de gestión de tareas, chat interno y gestión de tareas en grupo, cuenta con una asistente virtual que incorpora inteligencia artificial, que le permite aprender en base a la actividad del usuario y la del equipo, de tal forma que incluso podrá llegar a sugerir cual es la persona más adecuada para ocuparse de cada tarea programada y generar un análisis del tiempo estimado de la duración de un proyecto.

- Gmail: Es el correo electrónico más utilizado para la comunicación con los clientes y colaboradores empresariales por su fácil utilización; incluso permite la obtención de un dominio y una denominación de una marca específica previo el pago en una cuenta G.Suite.

- DropBox: Es perfecta para compartir archivos y carpetas, comparte de forma inmediata y con acceso instantáneo desde cualquier dispositivo para un usuario en particular o un grupo específico y da solución a problemas de almacenamiento de información. 
Para conseguir que las empresas alcancen sus objetivos, sean altamente competitivas y rentables, la comunicación es vital como estrategia empresarial por lo que es necesario dedicarle una atención especial (Eliorraga G; 2017). El determinar las herramientas utilizadas para la comunicación en el sector empresarial de la ciudad de Riobamba permite conocer sus preferencias tecnológicas y sus usos y beneficios.

\section{Metodología}

Se utilizó una metodología de carácter exploratoria, se tomó como universo los 724 contribuyentes especiales en estado activo que constan en el catastro del Registro Único de Contribuyentes del Sistema de Rentas Internas a Noviembre 2018 (RUC, 2018), el cálculo de la muestra fue de 336 empresas, con un margen de error del $4 \%$, tanto para $p$ y $q$ se consideró un valor de 0.5 (INEC, 2019).; la muestra es probabilística y se la realizó a través de un muestreo aleatorio simple sin repetición. La recolección de información fue con un equipo de cuatro personas, en varios días y diferentes sectores de la ciudad.

Inicialmente el cuestionario estuvo conformado por variables de clasificación con un total de 24 preguntas con base a los objetivos de la investigación. Después, con la participación de estadísticos expertos se realizó la validación de la encuesta, para indicar si la cuantificación fue exacta, y en base a sus recomendaciones se desecharon algunas preguntas y se reformularon otras, procediendo a la aplicación de una encuesta piloto con 17 interrogantes; y a realizar el análisis de aquellas consideradas más relevantes (Balarezo \& Cerón, 2014).

Se utilizó el coeficiente Alfa de Cronbach, en el software SPSS versión 22, donde se determinó un valor de 0.822 , por lo tanto, el nivel de confiabilidad es muy bueno ya que es mayor a 0.7 según el criterio general de pruebas de confiabilidad. (Domínguez-Lara, S. A., \& Merino-Soto, C., 2015)

La presentación de la información se la hizo de forma resumida mediante tablas de contingencia, conocidas también como de doble entrada, para el análisis de variables cualitativas nominales. Para la consolidación de los datos obtenidos de la aplicación de las encuestas se utilizó una variable sumatoria para todos los ítems y respuestas de cada uno de los participantes, a continuación, se procedió a caracterizar las sumatorias en función del uso de las herramientas tecnológicas utilizadas por las empresas para sus usos y beneficios.

\section{Resultados}

Una vez recopilada la información en base a las encuestas se procedió a la tabulación e interpretación de los resultados obtenidos, con el empleo del software estadístico SPSS.

En la tabla 1 referente al tipo de empresa se puede apreciar que el 57.70\% de las entidades participantes son públicas mientras que el $42.30 \%$ corresponden al sector privado. El $48.80 \%$ de 
ISSN: 2600-5859

los encuestados corresponden al sexo Femenino y el $51.20 \%$ al sexo masculino como se muestra en la tabla siguiente:

Tabla. 1: Tipo de Empresa

\begin{tabular}{llccc}
\hline & & \multicolumn{2}{c}{ Sexo } & \multirow{2}{*}{ Total } \\
\cline { 3 - 4 } & & Femenino & Masculino & \\
\hline \multirow{2}{*}{$\begin{array}{l}\text { Tú- } \\
\text { presa }\end{array}$} & $\begin{array}{l}\text { búca } \\
\text { blica }\end{array}$ & 99 & 95 & 194 \\
Total & $\begin{array}{l}\text { Pri- } \\
\text { vada }\end{array}$ & 65 & 77 & 142 \\
\hline
\end{tabular}

\section{Fuente: Elaboración propia}

La tabla 2 y la figura 1 muestran los resultados obtenidos en las empresas encuestadas con respecto a la aplicación más utilizada. Según los resultados obtenidos Wahtsapp ocupa el primer puesto, siendo la aplicación mayormente aceptada en las empresas con un total del $51.80 \%$, de los cuales el mayor porcentaje de utilización pertenece al sector público, le sigue Facebook que tiene un total del 33.00\%, Dropbox muestra un total del 14.60\%, Facebook Pages tiene un total del $0.3 \%$ al igual que Whatsapp Business, los dos casos corresponden a empresas privadas.

Tabla 2: La aplicación móvil que más utiliza su empresa

\begin{tabular}{|c|c|c|c|c|}
\hline & & \multicolumn{2}{|c|}{ Tipo de Empresa } & \multirow{2}{*}{ Total } \\
\hline & & Pública & Privada & \\
\hline \multirow{5}{*}{$\begin{array}{l}\text { Seleccione cual es la } \\
\text { aplicación móvil que } \\
\text { más utiliza su em- } \\
\text { presa }\end{array}$} & $\begin{array}{l}\text { Whatsapp Bus- } \\
\text { sines }\end{array}$ & 0 & 1 & 1 \\
\hline & Facebook Pages & 0 & 1 & 1 \\
\hline & Drobox & 28 & 21 & 49 \\
\hline & Facebook & 64 & 47 & 111 \\
\hline & Whatsapp & 102 & 72 & 174 \\
\hline Total & & 194 & 142 & 336 \\
\hline
\end{tabular}

\section{Fuente: Elaboración propia}


Figura 1: Aplicación Móvil más utilizada en las empresas

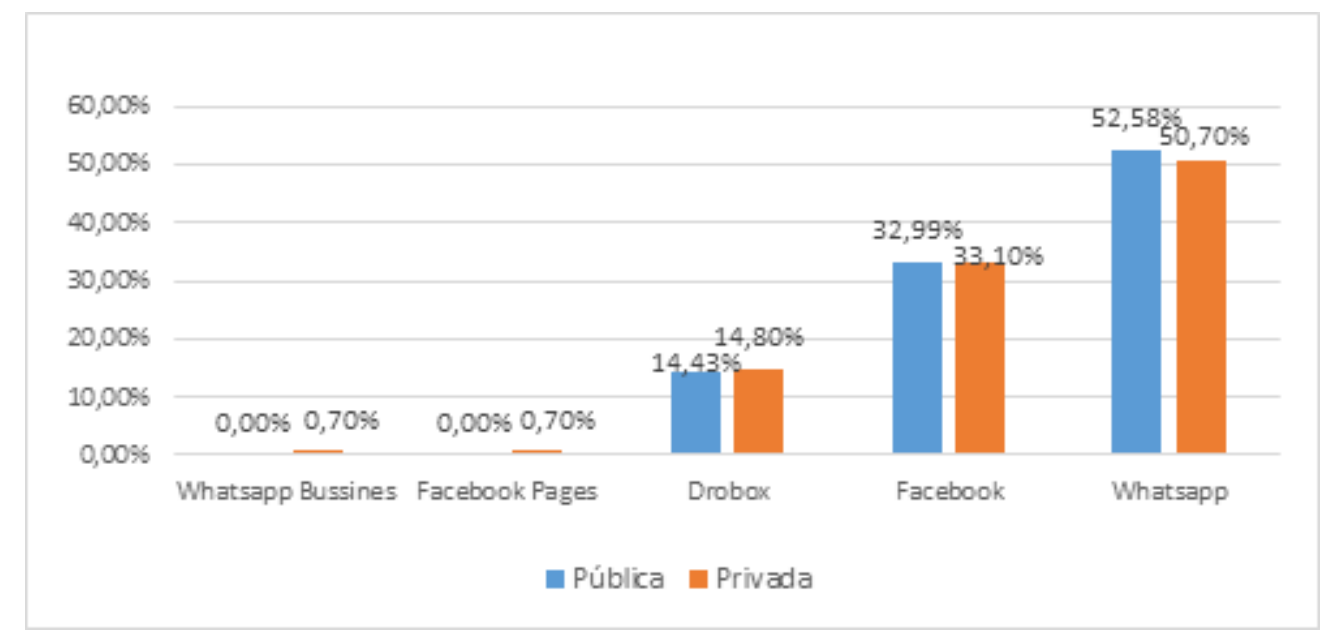

Fuente: Elaboración propia

En la tabla 3 y la figura 2 se puede apreciar las diversas actividades para las cuales las empresas utilizan las aplicaciones móviles; siendo la primera de ellas la comunicación interna con un total de $57.10 \%$; las actividades de promoción y publicidad tiene el $13.5 \%$ en los dos sectores encuestados, la comunicación externa el $6.60 \%$, la compartición de documentos muestra el 2.1\%, y el ítem todas las opciones el 19.6\%.

Tabla 3: Principal uso de las Apps dentro de su empresa

\begin{tabular}{llccc}
\hline & & \multicolumn{2}{c}{ Tipo de Empresa } & Total \\
\cline { 3 - 4 } & Compartición de documentos & 3 & 4 & 7 \\
& Comunicación Externa & 9 & 13 & 22 \\
$\begin{array}{l}\text { ¿Cuál es el principal } \\
\begin{array}{l}\text { uso de las Apps den- } \\
\text { tro de su empresa? }\end{array}\end{array}$ & Promoción y Publicidad & 22 & 27 & 49 \\
& Comunicación Interna & 120 & 72 & 192 \\
Total & Todas las opciones & 40 & 26 & 66 \\
\hline
\end{tabular}

Fuente: Software SPSS 
ISSN: 2600-5859

Figura 2: Principal uso de las apps en las empresas

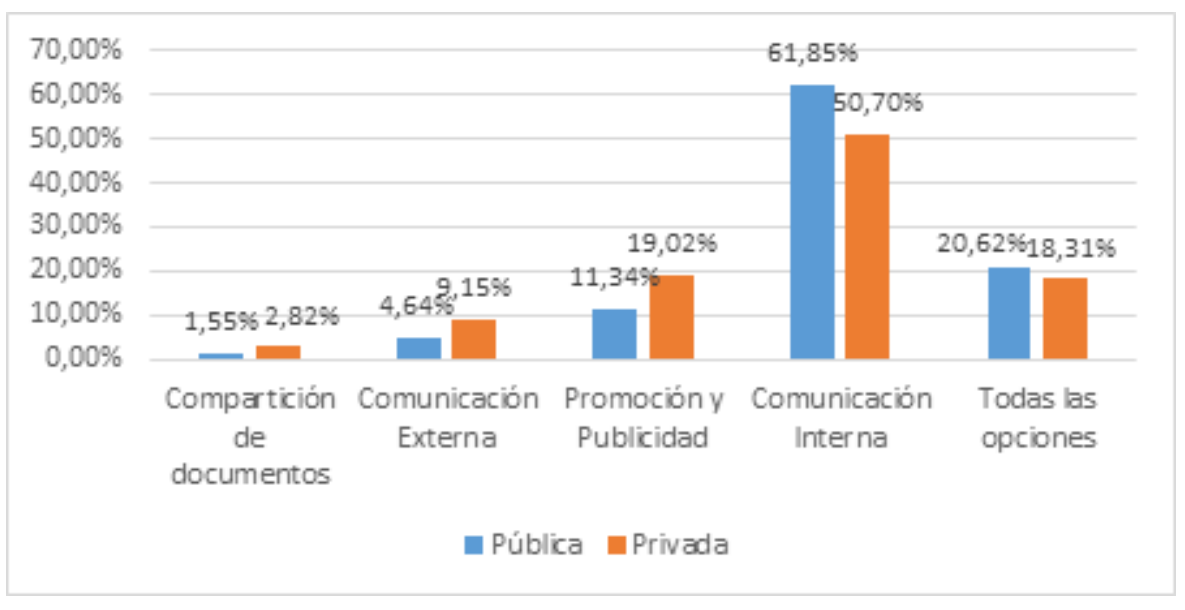

Fuente: Elaboración propia

La tabla 4 y figura 3 muestran que, dentro de los beneficios del uso de las aplicaciones móviles en las empresas, el nivel de pertinencia de los empleados tiene un total del $36.30 \%$ en las empresas encuestadas, la difusión de productos o servicios presenta el 28.50\%; el fomentar la fidelidad de los clientes o usuarios tiene el $13,10 \%$, la versatilidad para optimizar las compras presenta el 11.9\%; el ítem todas las opciones muestra un total del $10.1 \%$.

Tabla 4: Beneficios del uso de las Apps dentro de su empresa

\begin{tabular}{|c|c|c|c|c|}
\hline & \multicolumn{2}{|c|}{ Tipo de Empresa } & \multirow{2}{*}{ Total } \\
\hline & & Pública & Privada & \\
\hline \multirow{5}{*}{$\begin{array}{l}\text { ¿Cuál es el beneficio } \\
\text { más importante del } \\
\text { uso de las Apps den- } \\
\text { tro de su empresa? }\end{array}$} & $\begin{array}{l}\text { Fácil difusión de productos o servi- } \\
\text { cios }\end{array}$ & 66 & 30 & 96 \\
\hline & $\begin{array}{l}\text { Fomentar la fidelidad de los clientes } \\
\text { o usuarios }\end{array}$ & 18 & 26 & 44 \\
\hline & $\begin{array}{l}\text { Mayor nivel de pertinencia de los } \\
\text { empleados de la empresa }\end{array}$ & 78 & 44 & 122 \\
\hline & Versatilidad para optimizar compras & 13 & 27 & 40 \\
\hline & Todas las opciones & 19 & 15 & 34 \\
\hline Total & & 194 & 142 & 336 \\
\hline
\end{tabular}

Fuente: Elaboración propia 
ISSN: 2600-5859

Figura 3: Beneficios de las apps en las empresas

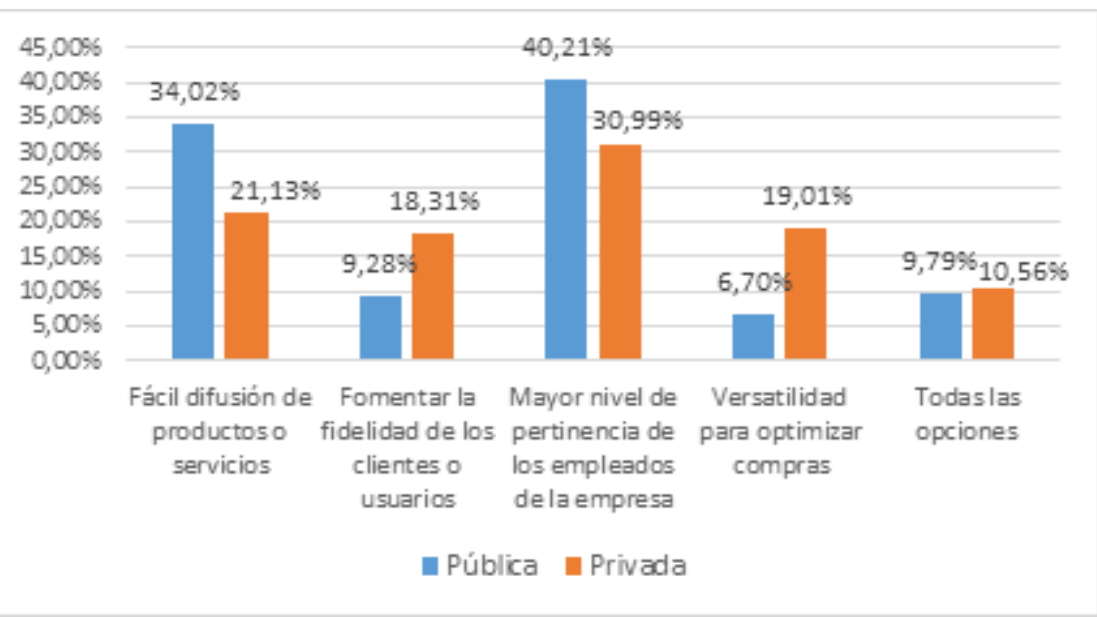

Fuente: Elaboración propia

La tabla 5 y la figura 4 permiten observar los beneficios según las empresas consideran más relevantes para sus clientes. La comunicación directa posee el $27.1 \%$ en las empresas encuestadas, el conocer nuevos productos o servicios muestra un $28.6 \%$, el ahorro de tiempo presenta un $17.3 \%$, la simplificación en trámites y/o procesos de negocios muestra un 15.5\%, el ítem todas las opciones tiene un porcentaje del $11.9 \%$.

Tabla 5: Beneficio para los clientes al usar Apps

\begin{tabular}{llccc}
\hline & & \multicolumn{2}{c}{ Tipo de Empresa } & \multirow{2}{*}{ Total } \\
\cline { 3 - 4 } & & Pública & Privada & \\
\hline \multirow{2}{*}{$\begin{array}{l}\text { ¿Cuál considera el } \\
\text { mayor beneficio } \\
\text { para sus clientes al }\end{array}$} & $\begin{array}{l}\text { Simplificación en trámites y/o pro- } \\
\text { usar Apps en su em- }\end{array}$ & 35 & 23 & 58 \\
presa? & Cesos de negocios & 32 & 20 & 52 \\
& Comocer los productos o servicios & 48 & 47 & 95 \\
Total & Todas las opciones & 60 & 31 & 91 \\
\hline
\end{tabular}

Fuente: Elaboración propia 
Figura 4: Beneficios de uso de las apps para los clientes

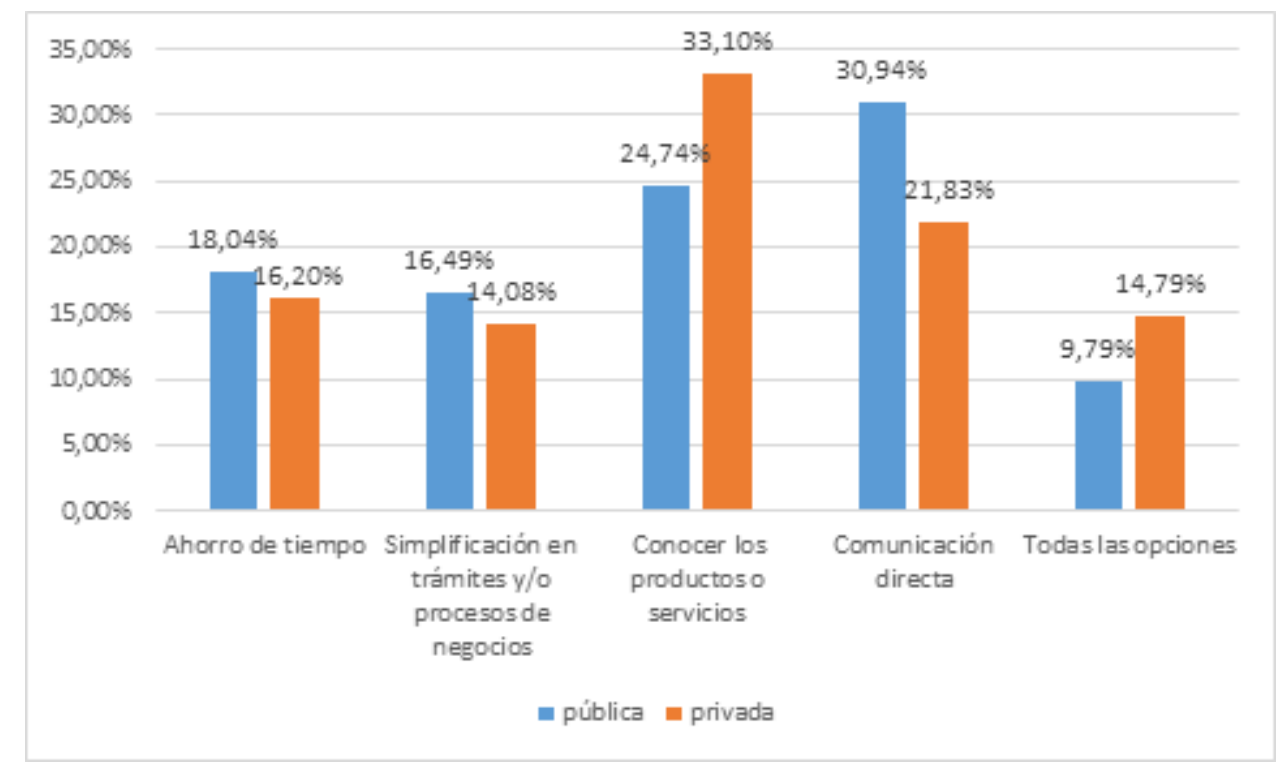

Fuente: Elaboración propia

\section{Discusión}

Las personas designadas por las empresas para la participación en la presente investigación fueron en su mayoría de sexo masculino, sin embargo, en las empresas públicas en su mayoría fueron mujeres.

La aplicación móvil mayormente utilizada en las empresas independientemente si es pública o privada es WhatssApp, seguido de Facebook y DropBox, al final se encuentran WhatssApp Bussines y Facebook Pages que sólo son utilizadas en las empresas privadas.

Los principales usos de las aplicaciones móviles en las diferentes empresas son (CEPAL, 2018):

- Como medio viable para la comunicación interna,

- El parámetro todas las opciones se ubica en el segundo puesto,

- Seguido de las actividades de promoción y publicidad de productos y/o servicios para los clientes o usuarios,

- Cuarto la comunicación externa entre empresas

- Quinto para la compartición de documentos. 
ISSN: 2600-5859

Dentro de los beneficios indicados por las empresas públicas en el empleo de aplicaciones móviles, en el orden de mayor a menor importancia están (Ródríguez, 2015):

- Mayor nivel de pertinencia de los empleados.

- La facilidad y rapidez que prestan estas herramientas para la difusión de productos y/o servicios, convirtiéndolas en un apoyo eficiente en la realización de diversos procesos.

- Todas las opciones presentadas en la encuesta ocupan el tercer lugar.

- El fomento de la fidelidad de los clientes o usuarios hacia la empresa.

Ahora, las empresas privadas coinciden en el primero y segundo último lugar, pero las posiciones de tercer y cuarto puesto se encuentran invertidos respecto a las empresas públicas.

Desde el punto de vista para los clientes de los dos tipos de empresas encuestadas, los beneficios que presentan las aplicaciones móviles son: permite conocer los productos y/o servicios, facilita una comunicación directa, ahorro de tiempo, la simplificación de trámites y/o procesos de negocio y el ítem de todas las opciones está al final de la lista.

\section{Conclusiones.}

- Existe una tendencia en el uso de las aplicaciones móviles como herramienta de apoyo en las tareas y procesos cotidianos en las empresas tanto públicas como privadas de la cuidad de Riobamba, las cuales se enmarcan en las tendencias mundiales.

- Las aplicaciones móviles son un canal de comunicación instantáneo entre las empresas y sus clientes y/o usuarios desde cualquier lugar y en cualquier momento, lo que las hace muy atractivas, por su gran facilidad de uso, la simplificación en trámites o procesos, el ahorro de tiempo y especialmente debido a su bajo costo (Urrutia, 2000).

- La mayoría de las empresas encuestadas están utilizando aplicaciones móviles para realizar la publicidad de sus productos y/o servicios, debido a su versatilidad y alcance; esto gracias a que cada vez son más personas quienes utilizan teléfonos inteligentes, lo que permite establecer un canal directo con los clientes o usuarios de las organizaciones. Es importante señalar que las empresas no utilizan aplicaciones móviles en versiones empresariales, pese a que estas presentan mayores prestaciones a nivel organizacional y a bajos costos, la principal razón es el desconocimiento de los beneficios e inclusive de la existencia misma de estas aplicaciones, siendo así que en el sector público no se observa el uso de este tipo de versiones y en el sector privado su empleo es mínimo.

\section{Referencias bibliográficas.}

Álvarez, A., \& Lesta, L. (2011). Medición de los aportes de la gestión estratégica de comunicación interna a los objetivos de la organización. Palabra clave, 14(1), 11-30. 
ISSN: 2600-5859

Arni Consulting Gruop. (2017). Shaping the future together. Consultado 06 de octubre de 2019. Disponible en https://www.negocios-internacionales.net/2017/10/14/virtualidadcotidiana/

Balarezo Toro, B. D., \& DT Cerón, J. (2014). "La Comunicación Organizacional Interna y su incidencia en el Desarrollo Organizacional de la Empresa SAN MIGUEL DRIVE" (Bachelor's thesis, Universidad Técnica de Ambato. Facultad de Ciencias Administrativas. Carrera de Organización de Empresas.).

Campos J. Grupo Femxa (2015). La importancia de la comunicación en la empresa. Consultado 25 de Noviembre 2019. Disponible en https://www.grupofemxa.es/la-importancia-de-lacomunicacion-en-la-empresa/

Catastros del registro único de contribuyentes (RUC) (2019). Consultado 10 de octubre 2019. Disponible en http://www.sri.gob.ec/web/guest/catastros

Centro Europeo de Postgrado (CEUPE). (2019). Aplicaciones móviles: tipos, ventajas e inconvenientes. Consultado 10 de diciembre 2019. Disponible en https://www.ceupe.com/blog/aplicaciones-moviles-tipos-ventajas-e-inconvenientes.html

Comisión Económica para América Latina y el Caribe CEPAL. (2018) Tecnologías de la información y las comunicaciones (TIC). Consultado 15 de Noviembre de 2019. Disponible en https://www.cepal.org/es/temas/tecnologias-de-la-informacion-y-lascomunicaciones-tic

Domínguez -Lara, S. A., \& Merino-Soto, C. (2015). ¿Por qué es importante reportar los intervalos de confianza del coeficiente alfa de Cronbach?. RLCSNJ, 13(2).

Eliorraga G. EAE Business School. (2018). ¿Es importante la comunicación interna en una empresa?. Consultado 12 de Octubre 2019. Disponible en https://retosdirectivos.eae.es/es-importante-la-comunicacion-interna-en-una-empresa/.

Facchin J. (2018) Las 41 aplicaciones móviles para emprendedores y trabajadores feelance. Consultado 10 de Octubre de 2019. Disponible en https://josefacchin.com/aplicacionesmoviles-emprendedores-freelance/

Gómez J. Cerem International Bussines School (2017). Claves para una buena comunicación interna en la empresa. Consultado 15 de Noviembre de 2019. Disponible en https://www.cerem.ec/blog/claves-para-una-buena-comunicacion-interna-en-laempresa

Instituto Nacional de Estadística y Censo (2019). Directorio de Empresas y Establecimientos (DIEE) 2018. Consultado 28 de Octubre de 2019. Disponible en http://www.ecuadorencifras.gob.ec/directoriodeempresas/

Jiménez, I. E. L. (2012). El impacto de la tecnología en la comunicación empresarial: Reflexiones y análisis. Razón y palabra, 17(79).

Nicolini, C., \& Parodi, J. (2015, April). Comunicación interna en la Administración Pública española: Claves para innovar. INAP. 
ISSN: 2600-5859

Vol. 3, $\mathrm{N}^{\circ} 1.2$, p. 6-19, marzo, 2020

Ritacco V. Strategic Minds. (2015).

La aplicación móvil como medio de comunicación interna. Consultado 5 de Noviembre. Disponible en http://strategicminds-pr.com/blog/la-aplicacion-movil-como-medio-decomunicacion-interna/

Rodríguez A. G. (2015). Organización de Estados Iberoamericanos. (2015). Ciencia, Tecnología y Sociedad: una mirada desde la Educación en Tecnología. Revista Iberoamericana de Educación. Consultado 5 de Noviembre 2019. Disponible en https://rieoei.org/historico/oeivirt/rie18a05.htm

Urrutia, A. A. (2000). Comunicación en la empresa. La importancia de la información interna en la empresa. Revista Latina de comunicación social, 3(27), 0. 
ISSN: 2600-5859

Vol. 3, $\mathrm{N}^{\circ} 1.2$, p. 6-19, marzo, 2020

PARA CITAR EL ARTÍCULO INDEXADO.

Viteri Ojeda, J. C., Andrade Álvarez, C. E., Valencia Ortiz, N. P., \& Castro Viteri, C. A. (2020). Usos y beneficios de las aplicaciones móviles en las empresas de la ciudad de Riobamba. ConcienciaDigital, 3(1.2), 6-19. https://doi.org/10.33262/concienciadigital.v3i1.2.1165

\section{Ciencia}

El artículo que se publica es de exclusiva responsabilidad de los autores y no necesariamente reflejan el pensamiento de la Revista Conciencia Digital.

El artículo queda en propiedad de la revista y, por tanto, su publicación parcial y/o total en otro medio tiene que ser autorizado por el director de la Revista Conciencia Digital.
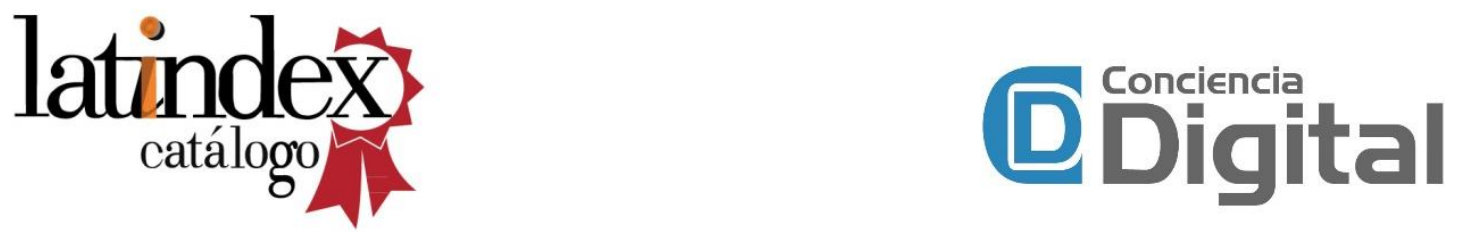